\title{
Design of an articulated robotic leg with nonlinear series elastic actuation
}

\section{Conference Paper}

Author(s):

Hutter, Marco (1); Remy, C. David; Siegwart, Roland

Publication date:

2009

Permanent link:

https://doi.org/10.3929/ethz-a-010034557

Rights / license:

In Copyright - Non-Commercial Use Permitted 


\title{
DESIGN OF AN ARTICULATED ROBOTIC LEG WITH NONLINEAR SERIES ELASTIC ACTUATION
}

\author{
MARCO HUTTER, C. DAVID REMY, ROLAND SIEGWART ${ }^{\dagger}$ \\ Autonomous Systems Lab, ETH Zurich, CLA E11.2, Tannenstrasse 3, 8092 Zurich, \\ Switzerland,mahutter@ethz.ch
}

\begin{abstract}
An articulated leg for the use in a running robot is presented. It is driven by series elastic actuation with a highly compliant spring at the knee joint to exploit periodic energy storage and passively support a running motion. The spring is connected with the knee motor by a cable pulley system, which allows the advantageous placement of the motor in the hip joint and enables us to use a compression spring instead of a heavier torsional element. Additionally, the pulley system creates a nonlinear spring characteristic at joint level which can be shaped by altering the cable tension. This nonlinearity and the inertial effects associated with it substantially increase the effective damping in the unloaded leg and allow precise foot-placement during the flight phase. Still, damping of the loaded leg during stance phase is kept minimal for highly efficient energy recovery.
\end{abstract}

\section{Introduction}

Human and animal running are based on an elastic oscillation of the main body on springy legs [1,2]. Energy is stored in muscles and tendons during the stance phase and recovered later in the gait cycle, which results in a very efficient motion $[1,3]$. When applying theses principles to robotics, it is often desirable that the stiffness of the elastic elements is low $[2,4]$. Higher compliances allow a longer stance phase with ample time to feed energy into the system and control posture. This reduces the power and bandwidth requirements for the actuators and minimizes peak forces, which in turn can help to significantly decrease the weight of the robot. To not impede the natural passive dynamic running motion, series elastic actuation $[5,6]$ can be used as an energy input and to reject disturbances. However, during flight, the spring severely impairs the control of joint-angles, which is critical for precise foot placement at touch down. This creates a trade-off with regard to the effective damping in the elastic element. High damping is desirable for more precise foot placement but leads to higher energy losses during stance, and vice versa.

\footnotetext{
Work partially supported by the Swiss National Science Foundation (SNF) (project 200021 119965/1).
} 


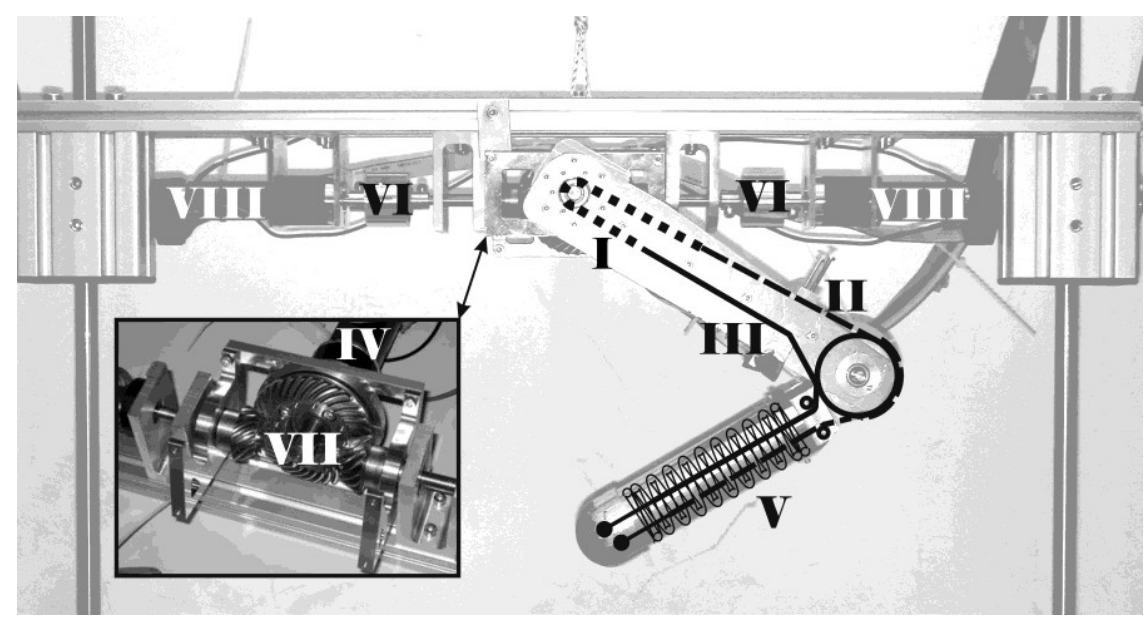

Figure 1. A miniature chain-drive (I) and two pulleys (II, III) connect the knee motor (IV) with a highly compliant spring (V) in the shank. The overlaid graphic illustrates the course of the pulleys for knee extension (II) and knee flexion (III). Elastic elements (VI) and a differential drive (VII) translate the action of the hip motors (VIII) into hip flexion/extension and abduction/adduction.

In this study, we present a prototype leg that utilizes a cable pulley system to engage a single linear compression spring that is only used uni-directional for knee flexion and extension. Variable pre-compression of this spring allows the creation of varying nonlinear spring characteristics. Additionally, the cable pulley system (in conjunction with a differential-drive hip-actuation) allows the placement of all actuators at the base of the leg, thus reducing the mass and inertia of thigh and shank, and improving the robot's running capabilities [7].

\section{Methods}

The robotic leg developed in this project (Figure 1) consists of three segments: main body, shank, and thigh. A differential drive (VII) translates the motion of the two hip motors (VIII) into flexion/extension and abduction/adduction of the hip. This allows us to place the hip motors directly at the main body. High performance elastomer-bushings are used as springs (VI) for series elastic actuation. The knee motor (IV) is placed directly at the hip joint. It is connected via a mini chain-drive (I), a cable pulley system (II, III), and a cable roll in the knee to the highly compliant knee spring (V) in the shank. As the cable pulleys for knee-flexion (III) and for knee-extension (II) are attached to the same side of the spring (Figure 2a), the spring is only used in one direction, namely compression. Variable pre-compressing of this system (by tensioning the pulley system) leads to different nonlinear spring characteristics (Figure 2b). 


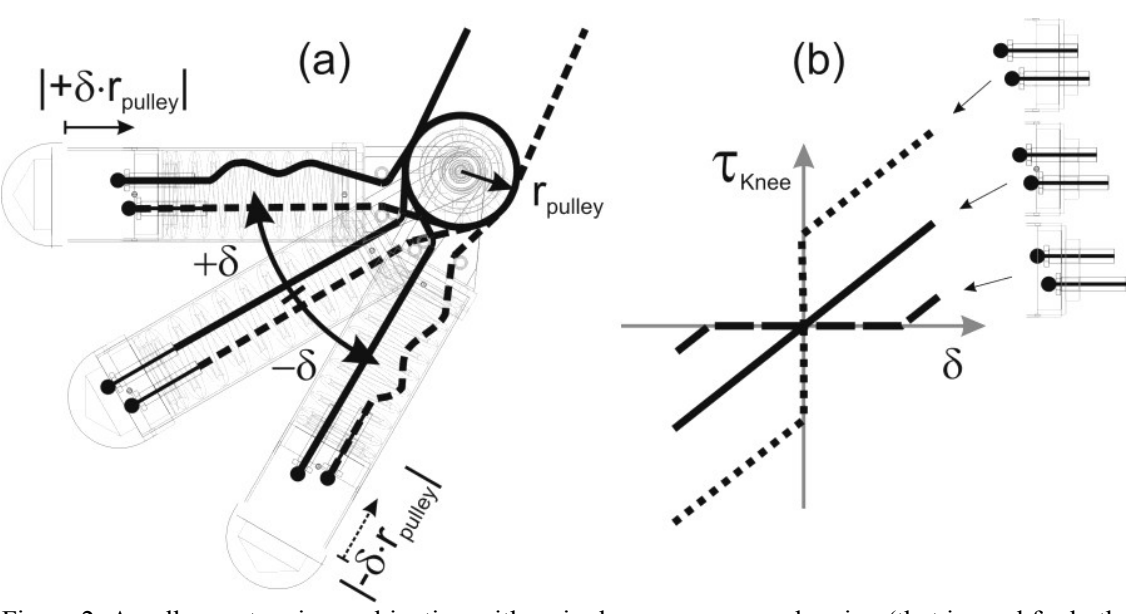

Figure 2. A pulley system in combination with a single pre-compressed spring (that is used for both, knee flexion and extension) results in a nonlinear spring-characteristic at joint level (a). By altering the pre-compression of the spring (which can be adjusted by tensioning the cable pulley) the spring characteristic at joint level can be changed (b).

Additionally to the static spring characteristic, dynamic effects must be considered since the mass of the spring largely accounts to the overall inertia of the shank. When the shank is crossing the neutral position, the direction of motion of the uni-directional spring instantaneously changes (Figure 3a) resulting in a mechanical collision in which energy is lost; a process that does not happen in a regular torsion spring that acts bi-directional (Figure $3 b$ ).

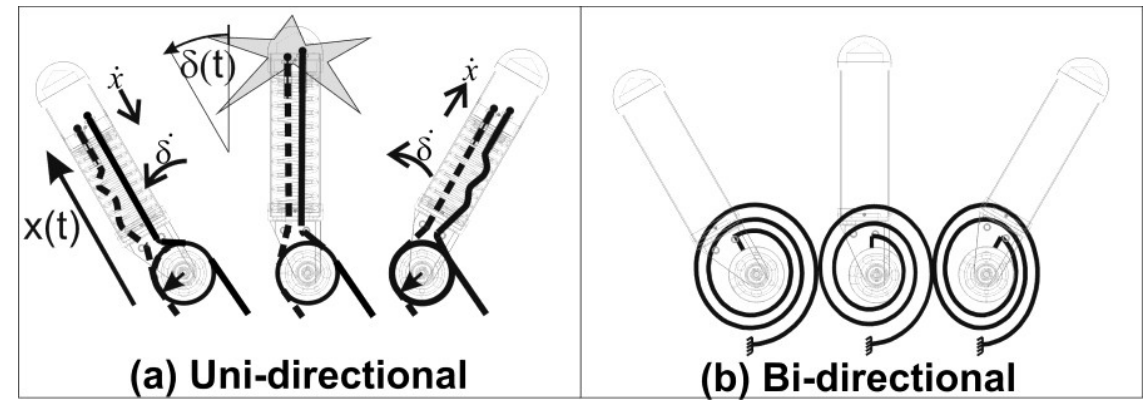

Figure 3. The uni-directional spring used in this study changes its direction of motion when the joint crosses the neutral position. This causes a collision in which energy is lost (a). This effect induces high damping in the unloaded joint. To obtain comparable dynamics, a bi-directional spring needs active damping to continuously dissipate energy (b). 


\section{Results}

The presented hardware is able to intrinsically solve the damping-dilemma introduced above. During the stance phase, the joint is loaded unilaterally, and the spring operates permanently in a linear region where no collisions occur. Energy storage and passive dynamic motion can be exploited efficiently without major losses due to unnecessary high damping. During flight, however, the unloaded joint will repeatedly pass the nonlinear region around the neutral position of the spring and energy is dissipated quickly.

The presented knee joint with torsional deflection $\delta$, overall inertia $M$ (rotating and translating masses) and differentiable force $h$ can be expressed as:

$$
M(\delta) \ddot{\delta}+h(\delta, \dot{\delta})=0
$$

(Note: The mass matrix $M$ depends additionally on the compression $x$ of the actual spring. Since this effect is comparably small, it was neglected for theoretical considerations. However, it was taken into account when performing the presented simulations). The kinematic relation between the deflection angle $\delta$ and the spring compression $x$ is given by the pulley radius $r$

$$
\dot{x}=-r \dot{\delta} \cdot \operatorname{sign}(\delta)
$$

Crossing the neutral position of the spring causes a reversion of the speed direction of the spring mass $m$ from the pre-impact velocity $\dot{x}^{-}$to the postimpact velocity $\dot{x}^{+}$that introduces an impulse in the cable:

$$
\Lambda=m \dot{x}^{+}-m \dot{x}^{-}=\left\{\begin{array}{l}
-m r\left(\dot{\delta}^{+}+\dot{\delta}^{-}\right) \stackrel{\text { from } \delta<0 \text { to } \delta>0}{ } \\
m r\left(\dot{\delta}^{+}+\dot{\delta}^{-}\right) \stackrel{\text { from } \delta>0 \text { to } \delta<0}{\longrightarrow}
\end{array}\right.
$$

Inserting into the integrated version of the equations of motion Eq. (1) results in:

$$
M\left(\dot{\delta}^{+}-\dot{\delta}^{-}\right)=\left\{\begin{array}{l}
r \Lambda \stackrel{\text { from } \delta<0 \text { to } \delta>0}{\stackrel{\text { from } \delta>0 \text { to } \delta<0}{\longrightarrow}} \\
-r \Lambda \stackrel{\text { fro }}{ }
\end{array}\right.
$$

Post-impact angular velocities are calculated from Eq. (3) and Eq. (4):

$$
\dot{\delta}^{+}=\frac{M-m r^{2}}{M+m r^{2}} \cdot \dot{\delta}^{-}
$$

The kinetic energy loss $\frac{T^{+}}{T^{-}}$of the collision can be expressed as 
(a) return map

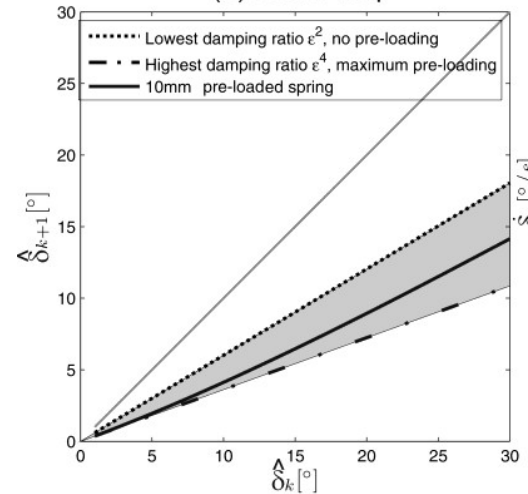

(b) phase portrait

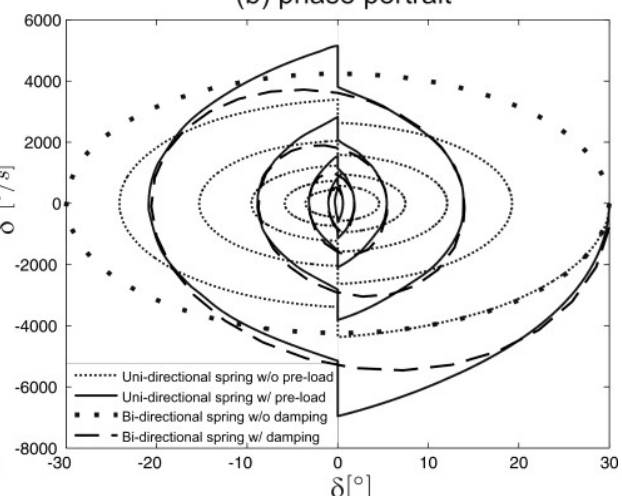

Figure 4. The amplitude ratio of two successive oscillation periods is shown as a return map (a). The inertia of the spring and the shank determine the maximal $\left(\varepsilon^{4}\right)$ and minimal $\left(\varepsilon^{2}\right)$ damping ratio. The exact amount of amplitude decrease can be adjusted in this range (gray area) by varying the pre-compressing of the spring. As can be seen in the phase portrait (b), energy is lost only when the joint crosses its neutral position. Higher pre-compressing of the spring leads to higher velocities when crossing the neutral position which in turn leads to higher energy losses. During stance phase (gray area), a uni-directional spring w/o pre-compression shows the same behavior as an equivalent bi-directional spring w/o damping. However, such a bi-directional spring needs strong continuous damping to show the same overall decrease in deflection amplitude and is therefore less efficient when used in a running robot.

$$
T^{+}=1 / 2 M \dot{\delta}^{+2}=1 / 2 M\left(\varepsilon^{2} \dot{\delta}^{-2}\right)=\varepsilon^{2} T^{-}
$$

With the inertia correlation $\varepsilon=\frac{M-m r^{2}}{M+m r^{2}}<1$.

Neglecting additional (e.g.: viscous or frictional) damping in the spring, energy is conserved while $\delta \neq 0$. I.e. the kinetic energy $T_{k}$ at the zero-crossing is equal to the potential energy $V_{k}$ at maximal deflection $\hat{\delta}_{k}$ :

$$
T_{k}=1 / 2 M \dot{\delta}_{k}^{-2}=1 / 2 c r^{2} \hat{\delta}_{k}^{2}+c \bar{x}_{p} \cdot r \hat{\delta}_{k}=V_{k}
$$

$V_{k}$ is a function of the spring constant $c$, the pre-compression force $F_{P}=c \cdot \bar{x}_{P}$, and the angle deflection amplitude. As two collisions happen in one full period, the overall energy loss is described by:

$$
\frac{T_{k+1}}{T_{k}}=\frac{V_{k+1}}{V_{k}}=\frac{1 / 2 c r^{2} \hat{\delta}_{k+1}^{2}+c \bar{x}_{p} r \hat{\delta}_{k+1}}{1 / 2 c r^{2} \hat{\delta}_{k}^{2}+c \bar{x}_{p} r \hat{\delta}_{k}}=\varepsilon^{4}
$$

Depending on the pre-compression $\bar{x}_{P}$, the damping ratio $\hat{\delta}_{k+1} / \hat{\delta}_{k}$ can be varied in the range of maximum $\left(\varepsilon^{4}\right)$ and minimum damping ratio $\left(\varepsilon^{2}\right)$ (Figure $4 a$ ). 


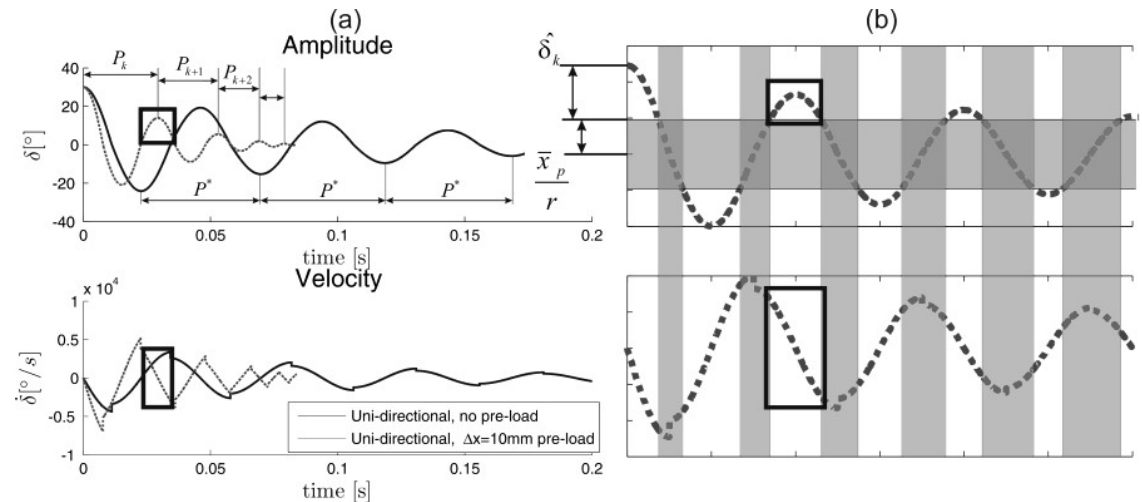

Figure 5. If the uni-directional spring is not pre-compressed, the amplitude decreases but the periodic time $P^{*}$ remains constant. Pre-compressing the spring increases the damping ratio and additionally reduces the periodic time as a function of amplitude (a). In comparison to a linear oscillation $\delta(t)=\left(\hat{\delta}_{k}+\Delta x / r\right) \sin (\omega t)$, pre-compressing the spring with $\bar{x}_{p} / r$ removes all states in which $\delta(t)<\bar{x}_{p} / r$. This is indicated in (b) by the gray area. This results in shorter periods and consequently higher damping per unit time.

Simulations of this mass-spring system confirm the theoretical considerations (Figure $4 \mathrm{~b}$ ). With the presented uni-directional spring design energy is only dissipated due to instantaneous velocity changes when crossing the neutral position of the joint. Furthermore, variation in the pre-compression of the spring can be used to adjust the damping ratio. During stance phase (gray area), when the energy should be fully recovered, no damping occurs. To achieve the same overall damping ratio with a regular torsion spring (dashed line), one would need continuous damping and hence lose energy during the stance phase.

Additionally to the increase of the damping ratio due to the internal collisions, the Eigenfrequency of a pre-compressed spring is growing when the oscillation amplitude is getting smaller. While damping over one cycle remains the same, cycles become shorter and the effective damping per time of the unloaded leg is substantially increased (Figure 5). The period time $P_{k}$ is directly related to the angle amplitude $\hat{\delta}_{k}$ and the pre-compression $\bar{x}_{P}$ :

$$
\frac{P_{k+1}}{P_{k}}=\frac{\pi-2 \arcsin \left(\bar{x}_{p} /\left(\bar{x}_{p}+r \hat{\delta}_{k+1}\right)\right)}{\pi-2 \arcsin \left(\bar{x}_{p} /\left(\bar{x}_{p}+r \hat{\delta}_{k}\right)\right)}
$$

Using Eq. (9) together with Eq. (8) results in a shortening of the period time that depends on the pre-compression and the inertia correlation $\varepsilon$. 
(a) Experiment

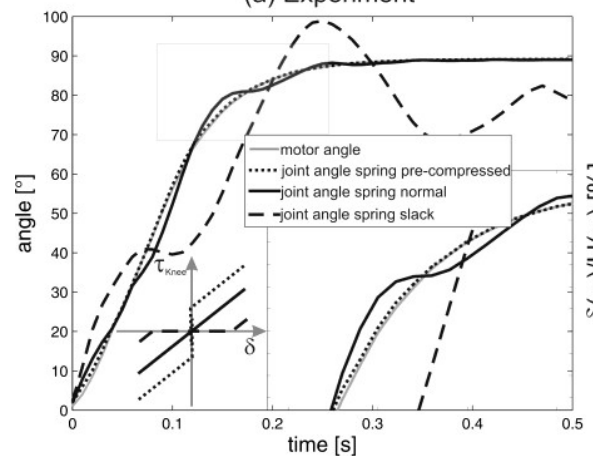

(b) Simulation

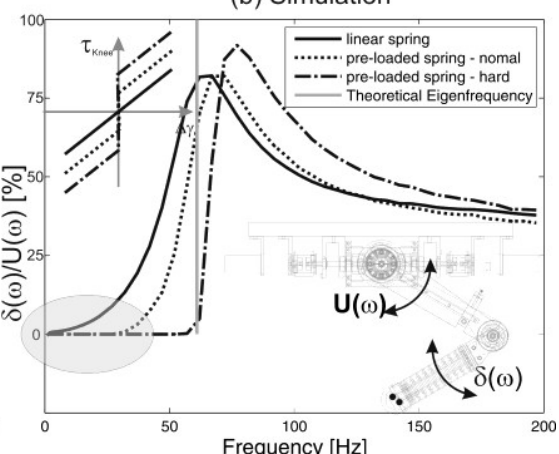

Figure 6. Pre-compressing the pulley system results in a nonlinear spring characteristic (dotted lines) of the knee joint that increases the effective damping around the neutral position. If the leg is not externally loaded, the knee angle follows the motor output exactly (a). This allows precise foot placement during the flight phase despite the elasticity in the leg. Unwanted oscillations, e.g. introduced by motion of the hip joint, are damped out completely in the frequency range utilized for locomotion. Shown in (b) is the frequency response of the knee joint as a function of hip excitation.

If a position demand step input is given to the knee motor of the unloaded leg (Figure 6a), the acceleration forces cause deflections when the spring is not precompressed. However, when the pre-compression force is high enough to counteract the inertial forces of the unloaded leg, no deflection can be observed. In the frequency range that is utilized for locomotion (Figure 6b), this effect makes it possible to perform precise foot placement purely open-loop; i.e. by controlling only the motor side of the series elastic actuator.

Successful open-loop 1D hopping experiments were performed using the presented hardware. Noteworthy is the excellent agreement of simulation and measurement, representing a final validation of the presented model (Figure 7). During flight phase, the leg position is controlled purely open-loop.

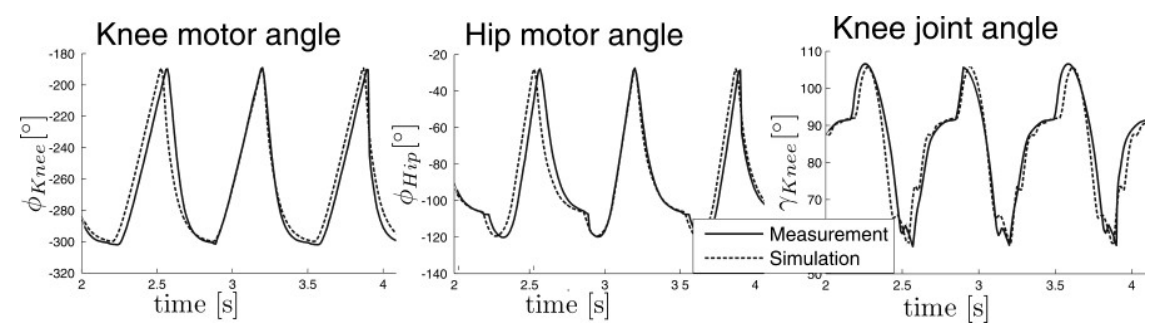

Figure 7. Motor and joint angles in a series of consecutive jumps show good agreement of simulation and measurement. Simulation and experiment are both started with the same initial conditions and utilize the same overall control strategy (velocity control during stance phase, position control during flight). 


\section{Discussion}

The use of a high compliant series elastic actuator at the knee reduced simulated energy consumption for steady state hopping by $21 \%$, and actuator power by $57 \%$, compared to a conventional system without elastic elements. The presented design that uses a uni-directional spring with an adjustable nonlinear spring characteristic offers the possibility to enable the natural dynamics during stance phase and suppress undesired deflection when the leg is unloaded, thereby solving an important issue in high-compliance locomotion. An energy efficient hopping motion that fully utilizes the passive dynamics of the elastic leg is achieved in simulations and experiments. The system is controlled completely open-loop, i.e. no information about the actual joint angle is required as the three effects discussed above will damp out unwanted deflections and force the unloaded joint to follow exactly. Furthermore, the compression spring used in this study has only half the weight of a comparable torsion spring, and offers the possibility of pre-compressing, which avoids problems with tolerances and backlash.

\section{Acknowledgments}

The authors gratefully acknowledge Jonas Fisler for his contribution in developing the prototype of the presented leg.

\section{References}

1. G. A. Cavagna, N. C. Heglund, and C. R. Taylor, "Mechanical work in terrestrial locomotion: two basic mechanisms for minimizing energy expenditure," AJP Regul Integr Comp Physiol, vol. 233, pp. 243-261, 1977.

2. C. Farley, J. Glasheen, and T. McMahon, "Running springs: speed and animal size," J Exp Biol, vol. 185, pp. 71-86, 1993.

3. M. H. Dickinson, et al., "How Animals Move: An Integrative View," Science, vol. 288, pp. 100-106, 2000.

4. A. Seyfarth, H. Geyer, M. Gunther, and R. Blickhan, "A movement criterion for running," Journal of Biomechanics, vol. 35, pp. 649-655, 2002.

5. G. Pratt and M. Williamson, "Series elastic actuators," in IROS, Pittsburg, PA, 1995, pp. 399-406.

6. D. W. Robinson, et al., "Series elastic actuator development for a biomimetic walking robot," in International Conference on Advanced Intelligent Mechatronics (AIM), Atlanta, USA, 1999, pp. 561-568.

7. R. M. Alexander, Principles of Animal Locomotion. Princeton, NJ: Princeton University Press, 2003. 